


\title{
NIKOLAUS KOPERNIKUS GESAMTAUSGABE
}

\author{
BEGRUNDET IM AUFTRAG \\ DER DEUTSCHEN FORSCHUNGSGEMEINSCHAFT \\ HERAUSGEGEBEN VON DER \\ KOPERNIKUS-KOMMISSION
}

BAND II

DE REVOLUTIONIBUS ORBIUM CAELESTIUM TEXTKRITISCHE AUSGABE

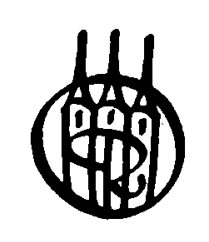

MÜNCHEN 1949

VERLAG VON R. OLDENBOURG 


\title{
NICOLAI COPERNICI THORUNENSIS
}

\author{
DE REVOLUTIONIBUS \\ ORBIUM CAELESTIUM \\ LIBRI SEX
}

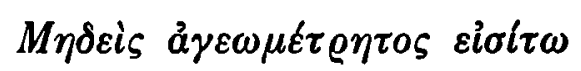

HANC EDITIONEM CURAVERUNT

FRANCISCUS ZELLER

CAROLUS ZELLER

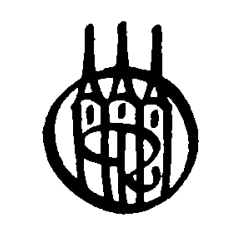

MDCCCCIL

IN AEDIBUS R. OLDENBOURG MONACHII 
VIRIS EMINENTISSIMIS QUI HAC TEMPORUM INIQUITATE PAGUM WURTTEMBERG-HOHENZOLLERN ADMINISTRANTES MAECENATUM MORE AD SUMPTUS HUIUS EDITIONIS LARGAM EX AERARIO PUBLICO CONTULERUNT STIPEM，DIGNA SIT GRATIARUM ACTIO 